\title{
Simple way to generate high order vacuum graphs
}

\author{
K. Kajantie* \\ Department of Physics, P.O. Box 64, FIN-00014 University of Helsinki, Finland \\ M. Laine ${ }^{\dagger}$ \\ Theory Division, CERN, CH-1211 Geneva 23, Switzerland \\ Y. Schröder ${ }^{\ddagger}$ \\ Department of Physics, P.O. Box 64, FIN-00014 University of Helsinki, Finland
}

(Received 13 September 2001; published 24 January 2002)

\begin{abstract}
We describe an efficient practical procedure for enumerating and regrouping vacuum Feynman graphs of a given order in perturbation theory. The method is based on a combination of Schwinger-Dyson equations and the two-particle-irreducible ("skeleton") expansion. The regrouping leads to skeletons containing only free propagators, together with "ring diagrams" containing all the self-energy insertions. As a consequence, relatively few diagrams need to be drawn and integrations carried out at any single stage of the computation and, in low dimensions, overlapping ultraviolet and infrared subdivergences can be cleanly isolated. As an illustration we enumerate the graphs contributing to the four-loop free energy in QCD, explicitly in a continuum and more compactly in a lattice regularization.
\end{abstract}

DOI: 10.1103/PhysRevD.65.045008

PACS number(s): 11.15.Bt, 11.10.Wx, 12.38.Bx

\section{INTRODUCTION}

There are many physics contexts where multiloop Feynman diagram computations are carried out. In QED one goes up to the four-loop level (for reviews see, e.g., [1]) because experiments are so precise. In particle physics phenomenology, particularly QCD, one goes up to the four-loop level (see, e.g., [2]) because the coupling constant is not small. In studying critical phenomena in the simplest $\mathrm{O}(N)$ condensed matter systems, one goes up to the five-loop level (see, e.g., [3]) because the effective expansion parameter is not small.

Studies of QCD at a finite temperature $T$ are faced with a similar challenge. Indeed, the coupling constant expansion converges even worse than at zero temperature requiring at least $T \gg 10^{3} \Lambda_{\mathrm{QCD}}$ to make any sense at all [4,5]. So far, though, only the resummed three-loop level has been reached for the simplest physical observable, the free energy [6], because a broken Lorentz symmetry makes the analysis much more complicated than in the cases mentioned above. In fact, even in principle only one more order is (partly) computable, and then the expansion breaks down completely [7]. Multiloop computations are not useless, though: the infrared problems can be isolated to a simple three-dimensional (3D) effective field theory [8] and studied nonperturbatively there [9], but to convert the results to physical units from lattice regularization still necessitates a number of fixed-order perturbative computations $[10,11,12]$.

As the loop order increases, so does the computational effort. The sheer enumeration of various diagrams and their symmetry factors becomes nontrivial. The group-theoretic and Lorentz structures of single graphs are involved. Finally,

\footnotetext{
*Email address: keijo.kajantie@helsinki.fi

†Email address: mikko.laine@cern.ch

"Email address: york.schroder@helsinki.fi
}

the scalar integrals remaining are hard to evaluate analytically. It is therefore clear that, ideally, one would like to automatize the whole procedure (for a review of the current status see, e.g., [13]).

In this paper we concentrate on the first step of any multiloop computation, the enumeration of various Feynman diagrams. This step should be the easiest to automatize, since all one needs is a straightforward evaluation of Wick contractions. Indeed, various packages, such as FEYNARTS [14] and QGRAF [15], are available for determining $n$-point functions in a given particle physics model.

For vacuum graphs in condensed matter systems a similar approach is possible. For the quartic $\mathrm{O}(N)$ scalar model the combinatorics is not yet too hard, but variants thereof already require some work. Consequently, graphical algorithms have been developed at four-loop order and beyond for a number of simple models [16].

In many cases, though, a straightforward generation of the full set of diagrams of a given loop order may not be the ideal way to go. In realistic theories there are very many graphs, and all integrals would have to be evaluated on the same footing. This is almost impossible, particularly if many different masses appear.

Here we wish to present what would seem to us to be a maximally manageable setup. All vacuum graphs are generated, but they are cleanly separated into two groups: one, of two-particle-irreducible (2PI) "skeletons" with free propagators, and the other, of "ring diagrams" with various selfenergy insertions (see also [17]). The self-energies, in turn, are directly obtained from lower order skeletons. We find that this setup economizes the generation of the various graphs quite significantly. We also point out that in low dimensions, relevant for statistical physics applications, the integrations remaining are qualitatively different in the two sets.

As an illustration of the setup, we enumerate the diagrams 
contributing to the four-loop free energy of finite temperature QCD (as well as QED and the symmetric phases of the electroweak theory and scalar electrodynamics). We hope, though, that the setup may be applicable to some other cases as well. That is why we wish to separate it from the evaluation of the integrals arising in the finite $T$ context [18], specific for that physical situation.

Our plan is the following. We summarize our basic notation in Sec. II, reorganize the standard skeleton expansion in Sec. III, review the Schwinger-Dyson equations for $n$-point and vacuum graphs in Sec. IV, and combine them with the modified skeleton expansion to obtain a generating formula for skeleton diagrams in Sec. V. The corresponding results are given for a lattice regularization of a generic model in Sec. VI. As an illustration, we show the loop expansion for the free energy of QCD and related models in Sec. VII. We discuss some basic properties of our setup and conclude in Sec. VIII.

\section{NOTATION}

Let us start by introducing a concise notation. While the method is valid for any theory, we explicitly give all equations for a generic $\varphi^{3}+\varphi^{4}$ model. Later on we discuss more specific examples within this class, in particular QCD, as well as some extensions of this class. The generic class also includes the electroweak sector of the standard model, both in its symmetric and its spontaneously broken phase.

The partition function is defined as

$$
Z[J]=\int \mathcal{D} \varphi e^{S[\varphi]+J \varphi},
$$

where $S[\varphi]$ is the action,

$$
S[\varphi]=-\frac{1}{2} \varphi_{i} \Delta_{i j}^{-1} \varphi_{j}+\frac{1}{3 !} \gamma_{i j k} \varphi_{i} \varphi_{j} \varphi_{k}+\frac{1}{4 !} \gamma_{i j k l} \varphi_{i} \varphi_{j} \varphi_{k} \varphi_{l},
$$

and summations over various indices, numbering (real scalar) fields and their internal and spacetime structures, are implied. Two comments are in order. First, we will for the moment not display fermions explicitly. As far as vacuum graphs are concerned, they do not introduce any complications apart from the usual overall minus sign for each closed loop and can thus be introduced only at the end [19]. Second, one should notice that the sign conventions in Eqs. (1), (2) are such that in the case of Euclidean actions, $\gamma_{i j k l}$ is typically negative.

For a theory with a broken symmetry, the inverse free propagator $\Delta^{-1}$ and the couplings $\gamma_{i j k \ldots}$ are functions of the order parameter, but otherwise there are no essential complications. We return to this point in Sec. IV A.

The partition function $Z[J]$ in Eq. (1) is the generating functional for full Green's functions, $\Gamma_{n}^{\text {full }}=\left.\delta_{J}^{n} Z[J]\right|_{J=0}$. As usual, we define

$$
W[J]=\ln Z[J],
$$

the generating functional of connected Green's functions, $\Gamma_{n}^{\text {conn }}=\left.\delta_{J}^{n} W[J]\right|_{J=0}$. Finally, one can define the effective action via

$$
S_{\text {eff }}[\phi]=W[J]-\phi J, \quad \phi=\delta_{J} W[J],
$$

which generates 1PI Green's functions, $\Gamma_{n}^{1 \mathrm{PI}}$ $=\left.\delta_{\phi}^{n} S_{\text {eff }}[\phi]\right|_{\phi=0}$. Note, in particular, that $\delta_{\phi} S_{\text {eff }}[\phi]=-J$. The vacuum, or free energy $F$ (made dimensionless by a division with the temperature $T$ ), can be obtained from any of the generating functionals as

$$
F=-\ln Z[0]=-W[0]=-S_{\text {eff }}[0] .
$$

From the basic relations $\phi=\delta_{J} W[J], \delta_{\phi} S_{\text {eff }}[\phi]=-J$, it follows that

$$
\delta_{J}^{2} W[J] \delta_{\phi}^{2} S_{\mathrm{eff}}[\phi]=-1 .
$$

Defining, as usual, the "proper" self-energy by

$$
\delta_{\phi}^{2} S_{\text {eff }}[\phi] \equiv-\Delta^{-1}+\Pi,
$$

we see from Eq. (6) that $\delta_{J}^{2} W[J]$ is the full propagator:

$$
\delta_{J}^{2} W[J] \equiv D[\phi]=\frac{1}{\Delta^{-1}-\Pi} \equiv \Delta+\Delta \Pi \Delta+\Delta \Pi \Delta \Pi \Delta+\cdots .
$$

We shall use here the following notation for free and full propagators, the proper self-energy, as well as general 1PI vertices:

$$
\begin{aligned}
& \Delta=-\quad \text { (free propagator) } \\
& D=-=-+(\mathbb{O}-\ldots \text { (full propagator }) \\
& I I=--10--\quad \text { (proper self-energy, with legs "amputated"), } \\
& \Delta^{-1}=--\not \boldsymbol{p}^{-} \quad \text { (inverse free propagator, with legs amputated) } \\
& \delta_{\phi}^{n} S_{\text {eff }}=\underset{\substack{: \\
n}}{i} \text { (general amputated } 1 \mathrm{PI} \text { vertex) }
\end{aligned}
$$




\section{SKELETON EXPANSION WITH FREE PROPAGATORS}

We next review the skeleton expansion for the free energy $F[20,21]$ and modify it such that full propagators can be replaced with free propagators [17]. By a skeleton we mean a 2PI vacuum diagram: one that remains connected even if any two lines are cut. The skeleton expansion has been used as the starting point also in [17].

It can be shown $[20,21]$ that the loop expansion for Eq. (5) can be written as

$$
F[D]=\sum_{i} c_{i}\left(\operatorname{Tr} \ln D_{i}^{-1}+\operatorname{Tr} \Pi_{i}[D] D_{i}\right)-\Phi[D]
$$

where $i=\{$ bosons, fermions $\}, c_{\text {boson }}=1 / 2$, and $c_{\text {fermion }}=-1$. Here $\Phi[D]$ collects all 2PI vacuum diagrams. The full propagators $D_{i}$ are related to their corresponding selfenergies by $D^{-1}=\Delta^{-1}-\Pi[$ cf. Eq. (8)], where $\Delta$ are the free propagators. Both $F, \Pi$, and $\Phi$ can be regarded as functionals of the full propagators. The partition function has an extremal property, such that the variation of $F$ with respect to any of the full propagators vanishes [20,21,22], giving a relation between skeletons and self-energies:

$$
\delta_{D_{i}} \Phi[D]=c_{i} \Pi[D]
$$

Here we have introduced the implicit notation that whenever a term is multiplied by $c_{i}$, the $\Pi$ 's and $D$ 's following it are assumed to carry the same subscript. Pictorially, Eq. (15) corresponds to getting a self-energy by "cutting a propagator" in all possible ways in the set of vacuum skeletons. Hence knowing the skeletons alone provides full information.

In Eqs. (14), (15), it is the full propagators $D$ which appear in the skeleton graphs and self-energies. We would instead like to obtain skeletons with free propagators. As a first step in this direction, we expand $D$ in terms of the selfenergy insertions $\Pi[D], D=\Delta \Sigma_{n \geqslant 0}(\Pi \Delta)^{n}$, to get

$$
\begin{aligned}
F= & \sum_{i} c_{i} \operatorname{Tr}\left[\ln \Delta^{-1}+\sum_{n \geqslant 2}\left(1-\frac{1}{n}\right)(\Pi \Delta)^{n}\right] \\
& -\Phi\left[\Delta \sum_{n \geqslant 0}(\Pi \Delta)^{n}\right] .
\end{aligned}
$$

We then have to evaluate $\Pi[D]$.

To go forward more explicitly, we restrict ourselves to the five-loop level here. Let the subscript $n$ denote the loop order, and write $\Pi=\Sigma_{n \geqslant 1} \Pi_{n}$. It turns out that we need at most $\Pi_{3}$. In a straightforward way, we obtain

$$
\begin{aligned}
\Pi_{1} & =\Pi_{1}[\Delta] \equiv \Pi_{1}^{\mathrm{irr}}[\Delta] \\
\Pi_{2} & =\Pi_{2}^{\mathrm{irr}}[\Delta]+\left(\Pi_{1}^{\mathrm{irr}}\left[\Delta+\Delta \Pi_{1}^{\mathrm{irr}} \Delta\right]\right)_{2} \\
& \equiv \Pi_{2}^{\mathrm{irr}}[\Delta]+\Pi_{2}^{\mathrm{red}(1)}[\Delta]
\end{aligned}
$$

$$
\begin{aligned}
\Pi_{3}= & \Pi_{3}^{\mathrm{irr}}[\Delta]+\left(\Pi_{2}^{\mathrm{irr}}\left[\Delta+\Delta \Pi_{1}^{\mathrm{irr}} \Delta\right]\right)_{3} \\
& +\left(\Pi_{1}^{\mathrm{irr}}\left[\Delta+\Delta \Pi \Delta+\Delta \Pi_{1}^{\mathrm{irr}} \Delta \Pi_{1}^{\mathrm{irr}} \Delta\right]\right)_{3} \\
\equiv & \Pi_{3}^{\mathrm{irr}}[\Delta]+\Pi_{3}^{\mathrm{red}(1)}[\Delta]+\Pi_{3}^{\mathrm{red}(2)}[\Delta]
\end{aligned}
$$

where $\Pi_{n}^{\text {irr }}$ are $n$-loop 1PI graphs, while $\Pi_{n}^{\text {red }(m)}$ are obtained by cutting $m$ lines in a lower order $\Pi_{n}^{\text {irr }}[\Delta]$ and dressing them appropriately:

$$
\begin{aligned}
\Pi_{2}^{\mathrm{red}(1)}[\Delta]= & \left(\Delta \Pi_{1}^{\mathrm{irr}} \Delta\right)_{j} \delta_{\Delta_{j}} \Pi_{1}^{\mathrm{irr}}[\Delta], \\
\Pi_{3}^{\mathrm{red}(1)}[\Delta]= & \left(\Delta \Pi_{1}^{\mathrm{irr}} \Delta\right)_{j} \delta_{\Delta_{j}} \Pi_{2}^{\mathrm{irr}}[\Delta] \\
& +\left(\Delta \Pi_{2} \Delta+\Delta \Pi_{1}^{\mathrm{irr}} \Delta \Pi_{1}^{\mathrm{irr}} \Delta\right)_{j} \delta_{\Delta_{j}} \Pi_{1}^{\mathrm{irr}}[\Delta], \\
\Pi_{3}^{\operatorname{red}(2)}[\Delta]= & \frac{1}{2}\left(\Delta \Pi_{1}^{\mathrm{irr}} \Delta\right)_{j}\left(\Delta \Pi_{1}^{\mathrm{irr}} \Delta\right)_{k} \delta_{\Delta_{j}} \delta_{\Delta_{k}} \Pi_{1}^{\mathrm{irr}}[\Delta] .
\end{aligned}
$$

For the explicit diagrammatic characteristics of $\Pi_{2}^{\mathrm{red}(1)}$, see Sec. V B.

It is easy now to unfold the loop expansion also for $\Phi[D]=\Sigma_{n \geqslant 2} \Phi_{n}$, the last term in Eq. (16). Up to the fiveloop level, we can write

$$
\begin{aligned}
\left(\Phi_{2}[D]\right)_{n \leqslant 5}= & \left(\Phi _ { 2 } \left[\Delta+\Delta\left(\Pi_{1}+\Pi_{2}+\Pi_{3}\right) \Delta\right.\right. \\
& +\Delta\left(\Pi_{1}+\Pi_{2}\right) \Delta\left(\Pi_{1}+\Pi_{2}\right) \Delta \\
& \left.\left.+\Delta \Pi_{1} \Delta \Pi_{1} \Delta \Pi_{1} \Delta\right]\right)_{n \leqslant 5}, \\
\left(\Phi_{3}[D]\right)_{n \leqslant 5}= & \left(\Phi _ { 3 } \left[\Delta+\Delta\left(\Pi_{1}+\Pi_{2}\right) \Delta\right.\right. \\
& \left.\left.+\Delta \Pi_{1} \Delta \Pi_{1} \Delta\right]\right)_{n \leqslant 5}, \\
\left(\Phi_{4}[D]\right)_{n \leqslant 5}= & \left(\Phi_{4}\left[\Delta+\Delta \Pi_{1} \Delta\right]\right)_{n \leqslant 5}, \\
\left(\Phi_{5}[D]\right)_{n \leqslant 5}= & \Phi_{5}[\Delta],
\end{aligned}
$$

where the arguments are to be Taylor expanded, with first derivatives obeying [cf. the diagrammatic identity Eq. (15), evaluated with free propagators]

$$
\delta_{\Delta_{i}} \Phi_{n}[\Delta]=c_{i} \Pi_{n-1}^{\mathrm{irr}}[\Delta]
$$

and higher ones bringing back reducible self-energies, defined in Eqs. (20)-(22).

Inserting these expansions into Eq. (16), we finally get, up to the five-loop level, 


$$
\begin{aligned}
-F= & -\sum_{i} c_{i} \operatorname{Tr} \ln \Delta^{-1}+\Phi_{2}[\Delta]+\Phi_{3}[\Delta]+\sum_{i} c_{i} \operatorname{Tr}\left[\frac{1}{2}\left(\Delta \Pi_{1}\right)^{2}\right]+\Phi_{4}[\Delta]+\sum_{i} c_{i} \operatorname{Tr}\left[\frac{1}{3}\left(\Delta \Pi_{1}\right)^{3}\right. \\
& \left.+\Delta \Pi_{1} \Delta\left(\Pi_{2}^{\mathrm{red}}+\frac{1}{2} \Pi_{2}^{\mathrm{red}(1)}\right)\right]+\Phi_{5}[\Delta]+\sum_{i} c_{i} \operatorname{Tr}\left[\frac{1}{4}\left(\Delta \Pi_{1}\right)^{4}+\left(\Delta \Pi_{1}\right)^{2} \Delta\left(\Pi_{2}^{\mathrm{irr}}+\frac{1}{2} \Pi_{2}^{\mathrm{red}(1)}\right)\right. \\
& \left.+\frac{1}{2} \Delta \Pi_{2}^{\mathrm{irr}} \Delta\left(\Pi_{2}^{\mathrm{irr}}+\Pi_{2}^{\mathrm{red}(1)}\right)+\Delta \Pi_{1} \Delta\left(\Pi_{3}^{\mathrm{irr}}+\frac{1}{2} \Pi_{3}^{\mathrm{red}(1)}+\frac{1}{3} \Pi_{3}^{\mathrm{red}(2)}\right)\right]
\end{aligned}
$$

or, written diagrammatically (and denoting by $F_{0}$ the noninteracting result),

$$
\begin{aligned}
& -F=-F_{0}+\Phi_{2}[\Delta] \\
& +\left(\Phi_{3}[\Delta]+\sum_{i} c_{i}\left(\frac{1}{2} \oint\right)\right) \\
& +\left(\Phi_{4}[\Delta]+\sum_{i} c_{i}\left(\frac{1}{3} \text { (1) }+9+\frac{1}{2} \text { (1) }\right)\right.
\end{aligned}
$$

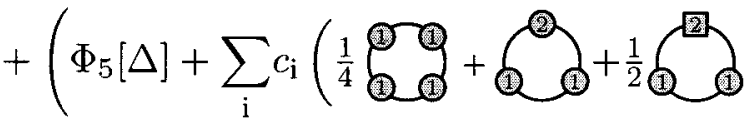

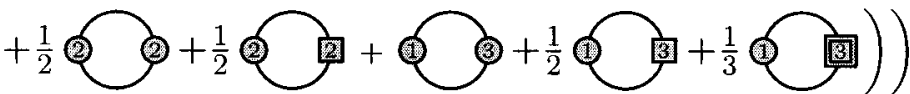

Here a circle with $n$ inside denotes $\Pi_{n}^{\text {irr }}$, a square $\Pi_{n}^{\text {red(1) }}$, and a double square $\Pi_{n}^{\operatorname{red}(2)}$. We will term the skeletons with free propagators, $\Phi_{n}[\Delta]$, irreducible. Note that the numerical factors in front of various types of ring diagrams do not appear to trivially follow from any simple symmetry argument (particularly in the case of reducible self-energy insertions), but are best worked out explicitly via the Taylor expansions we have described.

Equation (29) is the starting point of our setup. It expresses the free energy in an economic way in terms of the irreducible skeletons $\Phi_{n}[\Delta]$ : either as direct contributions or as self-energy insertions obtained from the same skeletons via Eqs. (27) and (20)-(22). We note that at the $n$-loop level, one needs $\Phi_{n}[\Delta]$, but only $\Pi_{n-2}[\Delta]$, obtained from $\Phi_{n-1}[\Delta]$.

\section{SCHWINGER-DYSON EQUATIONS WITH FULL PROPAGATORS}

Next, we need to generate the skeletons $\Phi_{n}[\Delta]$, needed in Sec. III. To do that, we first review briefly the general setup of Schwinger-Dyson (SD) equations, converted to our notation. The SD equations will then play a central role in our main result, Eq. (49), which is an explicit formula allowing for a systematic generation of all skeletons $\Phi_{n}[\Delta]$ —in principle to any order. In this section, we follow closely the very enjoyable presentation by Cvitanović [19].

\section{A. General $\boldsymbol{n}$-point functions}

The basic SD equation for the generating functional $Z[J]$ of full Green's functions derives from the trivial fact that the integral of a total derivative vanishes:

$$
0=\int \mathcal{D} \varphi \delta_{\varphi} e^{S[\varphi]+J \varphi}=\left(S^{\prime}\left[\delta_{J}\right]+J\right) Z[J] .
$$

For the generating functional of the connected Green's functions, Eq. (3), one gets

$$
0=S^{\prime}\left[W^{\prime}[J]+\delta_{J}\right]+J .
$$

Finally, for the effective action, Eq. (4), we use from Sec. II that $W^{\prime}[J]=\phi, \delta_{J}=(\delta \phi / \delta J) \delta_{\phi}=W^{\prime \prime}[J] \delta_{\phi}=D[\phi] \delta_{\phi}$, and $J=-S_{\text {eff }}^{\prime}[\phi]$ to obtain

$$
S_{\text {eff }}^{\prime}[\phi]=S^{\prime}\left[\phi+D[\phi] \delta_{\phi}\right] .
$$

Putting $\phi \rightarrow 0$ on the right-hand side, this gives the SD equation for the one-point function, while taking derivatives with respect to $\phi$ on both sides of Eq. (32) and putting $\phi \rightarrow 0$ only afterwards generates SD equations for higher-point Green's functions,

$$
\Gamma_{n}^{1 \mathrm{PI}}=\left.\delta_{\phi}^{n-1} S^{\prime}\left[\phi+D[\phi] \delta_{\phi}\right]\right|_{\phi=0} .
$$

Here $D[\phi]$ is in Eq. (8), and we note that

$$
\delta_{\phi} D[\phi]=D[\phi]\left(\delta_{\phi}^{3} S_{\text {eff }}[\phi]\right) D[\phi] .
$$

A note may be in order here concerning theories with spontaneously broken symmetries. In that case, $\phi$ corresponds to the fluctuating field around some reference value $v$, typically $v \equiv\langle\varphi\rangle$. The quantity we should ultimately be computing is the free energy density as a function of $v$ : i.e., the effective potential $V(v)=F /$ (volume). Then everything goes as before: we still put $\phi \rightarrow 0$ in the equa- 
tions above after differentiation, while the condensate $v$ appears as a parameter in the free propagators as well as in the cubic and quartic couplings in Eq. (2) [the term $J \varphi$ linear in $\varphi$ in Eq. (1) need not be changed [23]]. The graphs also remain the same: only 1 PI graphs, generated by the loop expansion in Eq. (29), are to be included [23]. Tadpole-type graphs often associated with broken symmetries would only be generated if we want to reexpand the value of $V(v)$ at the broken minimum in a strict loop expansion: writing $V$ $=\Sigma_{n \geqslant 0} V_{n}, v=\Sigma_{n \geqslant 0} v_{n}$, such that $V_{0}^{\prime}\left(v_{0}\right)=0$, implies

$$
\begin{aligned}
& \left.V(v)\right|_{V^{\prime}(v)=0}=V_{0}\left(v_{0}\right)+V_{1}\left(v_{0}\right)+\left[V_{2}-\frac{1}{2} \frac{\left(V_{1}^{\prime}\right)^{2}}{V_{0}^{\prime \prime}}\right]_{v=v_{0}} \\
& +\left[V_{3}-\frac{V_{1}^{\prime} V_{2}^{\prime}}{V_{0}^{\prime \prime}}+\frac{1}{2} \frac{\left(V_{1}^{\prime}\right)^{2} V_{1}^{\prime \prime}}{\left(V_{0}^{\prime \prime}\right)^{2}}-\frac{1}{6} \frac{\left(V_{1}^{\prime}\right)^{3} V_{0}^{\prime \prime \prime}}{\left(V_{0}^{\prime \prime}\right)^{3}}\right]_{v=v_{0}} \\
& +\left[V_{4}-\frac{1}{2} \frac{\left(V_{2}^{\prime}\right)^{2}+2 V_{1}^{\prime} V_{3}^{\prime}}{V_{0}^{\prime \prime}}+\frac{1}{2} \frac{2 V_{1}^{\prime} V_{2}^{\prime} V_{1}^{\prime \prime}+\left(V_{1}^{\prime}\right)^{2} V_{2}^{\prime \prime}}{\left(V_{0}^{\prime \prime}\right)^{2}}\right. \\
& -\frac{1}{6} \frac{3\left(V_{1}^{\prime}\right)^{2}\left(V_{1}^{\prime \prime}\right)^{2}+3\left(V_{1}^{\prime}\right)^{2} V_{2}^{\prime} V_{0}^{\prime \prime \prime}+\left(V_{1}^{\prime}\right)^{3} V_{1}^{\prime \prime \prime}}{\left(V_{0}^{\prime \prime}\right)^{3}}
\end{aligned}
$$

$$
\left.+\frac{1}{24} \frac{12\left(V_{1}^{\prime}\right)^{3} V_{1}^{\prime \prime} V_{0}^{\prime \prime \prime}+\left(V_{1}^{\prime}\right)^{4} V_{0}^{\prime \prime \prime \prime}}{\left(V_{0}^{\prime \prime}\right)^{4}}-\frac{1}{8} \frac{\left(V_{1}^{\prime}\right)^{4}\left(V_{0}^{\prime \prime \prime}\right)^{2}}{\left(V_{0}^{\prime \prime}\right)^{5}}\right]_{v=v_{0}}+\cdots
$$

where the latter terms inside the square brackets correspond to various tadpole graphs, with obvious notation: $1 / V_{0}^{\prime \prime}$ is the free propagator of the Higgs particle with a vanishing momentum, $V_{1}^{\prime}\left(V_{1}^{\prime \prime}\right)$ is a one-loop diagram with one leg (two legs), $V_{0}^{\prime \prime \prime}$ is a three-vertex, etc.

Let us now illustrate the structure of Eq. (33) for the generic model in Eq. (2). Starting from Eq. (2), writing down indices, and employing Eq. (34), we obtain, for the righthand side of Eq. (32),

$$
\begin{aligned}
\delta_{\phi_{i}} S= & -\Delta_{i j}^{-1} \phi_{j}+\frac{1}{2} \gamma_{i j k}\left(\phi_{j} \phi_{k}+D_{j k}\right)+\frac{1}{6} \gamma_{i j k l}\left(\phi_{j} \phi_{k} \phi_{l}\right. \\
& +D_{j k} \phi_{l}+D_{k l} \phi_{j}+D_{l j} \phi_{k} \\
& \left.+D_{j m} D_{k n} D_{l o} \delta_{\phi_{m}} \delta_{\phi_{n}} \delta_{\phi_{o}} S_{\text {eff }}[\phi]\right) .
\end{aligned}
$$

We now take further derivatives according to Eq. (33). Putting $\phi=0$ after each differentiation, we thus obtain the standard equations [written in the notation of Eqs. (9)-(13)]

$$
\text { - - }=
$$

where "cyclic $\left(n_{1}, n_{2}, \ldots\right)$ " denotes cyclic permutations of the legs numbered. We have not written down the two-loop terms in Eq. (41), since they are not needed in our explicit four-loop demonstration below. Likewise, all higher-point 1PI functions $\Gamma_{n}^{1 \mathrm{PI}}, n \geqslant 5$, start with one-loop graphs in the model of Eq. (2) and will again not contribute at this order; they will for $\Phi_{5}$, as well as in the model of
Sec. VI.

Let us stress that in a local theory the manipulations needed in Eq. (33) can essentially be made using regular derivatives and can thus easily be implemented algebraically. Introducing furthermore $\hbar$ as a loop counting parameter [24] allows for an iterative solution of the corresponding SD equations. 


\section{B. Vacuum diagrams}

The SD formalism above provides equations relating $n$-point Green's functions. To incorporate vacuum diagrams, one can use another simple trick: scaling. Noting that, e.g., $Z[J]$ is a functional of all interaction parameters present in the action, $Z\left[J, \gamma_{i j}, \gamma_{i j k}, \ldots\right]$, one can derive hosts of relations by varying any of these parameters.

A most useful example is to rescale the entire action as $S[\varphi] \rightarrow(1 / \hbar) S[\varphi]$ and then vary $\hbar:$

$$
-\hbar \partial_{\hbar} \ln Z[J]=\left\langle\frac{1}{\hbar} S[\varphi]\right\rangle=\frac{1}{Z[J]} \frac{1}{\hbar} S\left[\delta_{J}\right] Z[J] .
$$

Rewriting this in the "connected" language (recall $W=\ln Z$ ),

$$
-\hbar \partial_{\hbar} W[J]=\frac{1}{\hbar} S\left[W^{\prime}[J]+\delta_{J}\right],
$$

allows one to finally go over to 1PI functions $\left(\partial_{\hbar} W=\partial_{\hbar} S_{\text {eff }}\right.$ $+S_{\mathrm{eff}}^{\prime} \partial_{\hbar} \phi+J \partial_{\hbar} \phi=\partial_{\hbar} S_{\mathrm{eff}}, W^{\prime}=\phi$, and $\left.\delta_{J}=W^{\prime \prime} \delta_{\phi}=D[\phi] \delta_{\phi}\right)$ :

$$
-\hbar \partial_{\hbar} S_{\mathrm{eff}}[\phi]=\left\langle\frac{1}{\hbar} S[\varphi]\right\rangle=\frac{1}{\hbar} S\left[\phi+D[\phi] \delta_{\phi}\right]
$$

The free energy $F=-S_{\text {eff }}[0]$ can now be obtained by setting $\phi=0$ and integrating over $\hbar$.

Noting again that after a rescaling of the integration variables an expansion in $\hbar$ is equivalent to the loop expansion [24], one can integrate the left-hand side of Eq. (44) by $\int_{\hbar}(1 / \hbar)[\cdots]$, but on the right-hand side one integrates over the loop number. Writing

$$
-S_{\text {eff }}[0]=F=F_{0}+F_{\text {int }}=F_{0}+\sum_{n \geqslant 2} F_{n}^{\text {int }}
$$

where $n$ counts the number of loops, it follows that

$$
F_{n}^{\text {int }}=\frac{1}{n-1}\left\{\left.S\left[\phi+D[\phi] \delta_{\phi}\right]\right|_{\phi=0}\right\}_{n}, \quad n \geqslant 2 .
$$

Illustrating Eq. (46) for our generic theory in Eq. (2), we get

$$
F_{n}^{\mathrm{int}}=\frac{1}{n-1}\left\{-\frac{1}{2} \bigcirc+\frac{1}{6} \bigcirc+\frac{1}{8} \bigcirc+\frac{1}{8} \bigcirc+\frac{1}{24} \bigcirc\right\}_{n}
$$

where we again use the notation of Eqs. (9)-(13).

In principle the whole loop expansion can now be generated from Eq. (47), using Eqs. (38)-(41). The $n$-loop vacuum diagrams are expressed in terms of 1PI $n$-point functions, which in turn are governed by a set of SD equations. Looking closer at it, though, it is somewhat of a mess: one has to expand full propagators in terms of free ones and the $\Pi$ 's, use SD equations to iterate loops for $\Pi$ 's, which brings back full propagators, etc. Fortunately, none of this is necessary for Eq. (29), as we now explain.

\section{GENERATING THE IRREDUCIBLE SKELETONS $\Phi[\Delta]$}

The key observation for combining Schwinger-Dyson equations and the skeleton notation in a useful way is that we need to extract from Eq. (47) only a specific part $\Phi[\Delta]$ : we already know, by Eq. (29), what all the rest combines into. But then full propagators can be replaced by free propagators in all but the first term in Eq. (47). Indeed, any self-energy insertion within one of the other graphs leads to a two-particle-reducible (2PR) diagram. For the same reason, the 1PI vertices in Eq. (47) can be iterated by using the SD equations of the form in Eqs. (40), (41), but with free propagators. More precisely, it goes as follows.

To generate the irreducible skeletons $\Phi[\Delta]$ from Eq. (47), it is sufficient to expand the first term as

$$
\begin{aligned}
Q & =O+\bigcup^{0}+\{2 \mathrm{PR}\} \\
& =\operatorname{Tr} 1+\frac{1}{2} \bigcirc+\frac{1}{2} \bigcirc+\frac{1}{2} \bigcirc+\frac{1}{6} \bigcirc+\{2 \mathrm{PR}\},
\end{aligned}
$$

where in the second step Eq. (38) was used. Taking into account the minus sign in the relation of $F$ and $\Phi[\Delta]$ [cf. Eq. (29)] and writing again the loop expansion as $\Phi=\Sigma_{n \geqslant 2} \Phi_{n}$, one finally obtains a closed exact equation

$$
\Phi_{n}[\Delta]=\frac{1}{n-1}\left\{\frac{1}{12} \bigcirc+\frac{1}{8} \bigcirc+\frac{1}{8} \bigcirc 9+\frac{1}{24} \bigcirc\right\}_{n}, n \geq 2 .
$$

Equation (49) is our main result. It generates all skeletons of all orders in the theory of Eq. (2), once Eqs. (40), (41) are used (with free propagators). The skeletons, in turn, generate self-energies via Eq. (27) and the analogues of Eqs. (20)(22). Inserted finally into Eq. (29), we obtain the free energy $F$. 


\section{A. Vacuum skeletons up to the five-loop level}

The procedure of working out Eq. (49) is simple and mechanical and can, at least up to the four-loop level, even be carried out by hand, as we shall demonstrate. The only complication arising is the identification of equivalent topologies: the same graph can be written in very many different ways. In order to deal with this situation, it appears easiest to assign an algebraic notation for the different topologies, rather than a mere graphical one. For example, one can count the numbers of three-point and four-point vertices appearing in the graph, and within those equivalence classes, one can use a matrix notation for how the vertices are connected. The significant entries of the matrix can be ordered to a single number, and by doing the same for all possible orderings of the vertices, a unique representative (say, the smallest of such numbers) can be assigned to each topology. For an explicit implementation of this kind of a procedure, see the second paper in [16].

Let us now explicitly work out the diagram classes in Eq. (49) up to the four-loop level. For the first one, inserting Eq. (40) gives either a two-loop graph, or three-loop graphs to be iterated further on, or directly four-loop graphs:

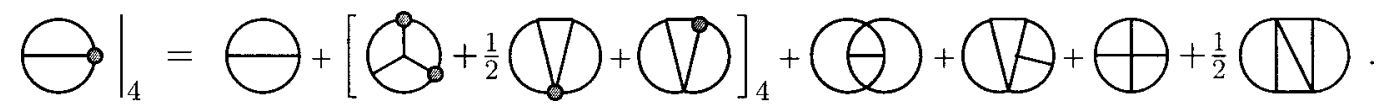

Here the further iterations give

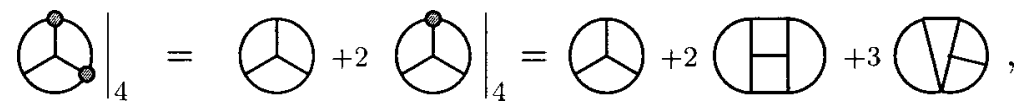

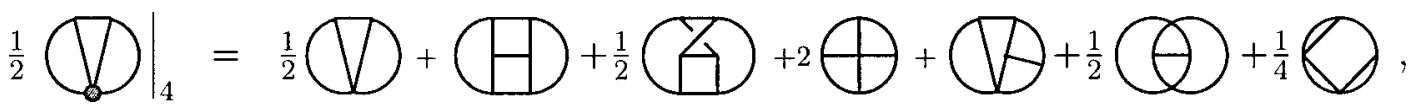

$$
\begin{aligned}
& \left.Q 9\right|_{4}=Q+\frac{1}{2} Q
\end{aligned}
$$

We have dropped five-point functions each time they appear, since in the model of Eq. (2), they start with a one-loop term, so that diagrams containing them generate higher loop orders.

The second class in Eq. (49) only contributes to $\Phi_{2}[\Delta]$ and is trivial. For the third class in Eq. (49),

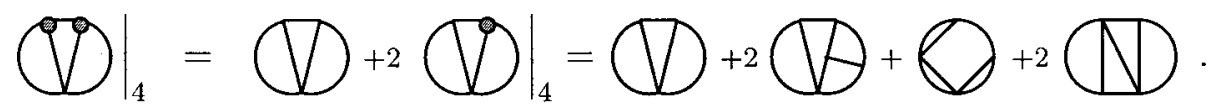

For the fourth class, we only need the one-loop terms in Eq. (41),

$$
(0)_{4}=0+3 \theta+\frac{3}{2}
$$

Collecting finally these different contributions together with coefficients according to Eq. (49), we get

$$
\Phi_{2}=\frac{1}{12} \longrightarrow+\frac{1}{8}
$$

Proceeding to higher loop orders, an automatized treatment proves essential, for the reasons outlined above. Implementing our generic formulas as well as an ordering algorithm separating topologies in FORM [25], we obtain in a straightforward way 
the complete set of five-loop skeletons,

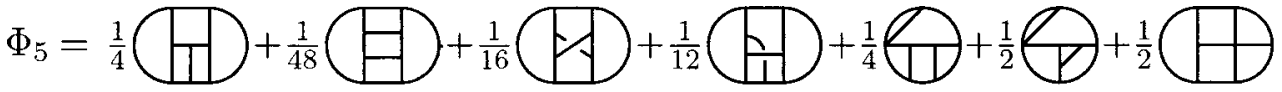

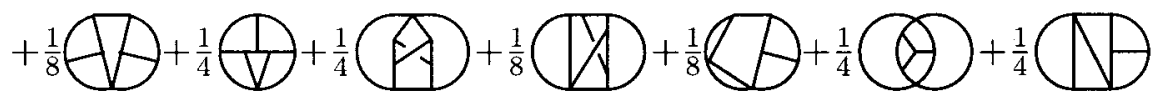

$$
\begin{aligned}
& +\frac{1}{8} \theta+\frac{1}{2} Q+\frac{1}{8} Q+\frac{1}{4} Q+\frac{1}{16} \square+\frac{1}{8}\left(\frac{1}{6}+\frac{1}{4}\right.
\end{aligned}
$$

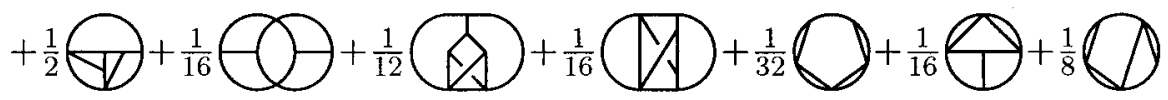

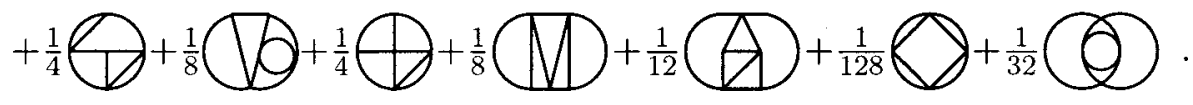

Note once more that these skeletons are all that is needed for generating the loop expansion for the full free energy, as discussed above.

\section{B. Self-energies up to the two-loop level}

Now that we have $\Phi_{n}[\Delta]$ in Eqs. (56) -(59), irreducible as well as reducible self-energies can easily be obtained with Eqs. (27), (20)-(22), etc. For bosonic particles, for instance $\left(c_{i}=\frac{1}{2}\right)$, we get

$$
\begin{aligned}
& \Pi_{1}^{\mathrm{irr}}=-\left(1-{ }^{-}=\frac{1}{2} \bigcirc-\frac{1}{2} \bigcirc\right.
\end{aligned}
$$

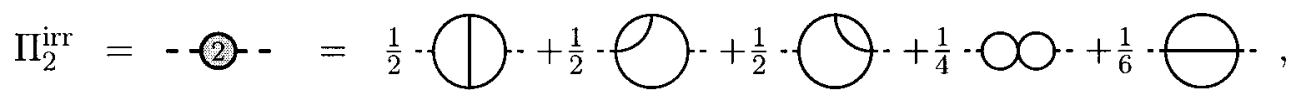

$$
\begin{aligned}
& \Pi_{2}^{\mathrm{red}(1)}=-2
\end{aligned}
$$

etc. Note that the outcome of the derivative in Eq. (27) must be symmetric in all (bosonic) indices. The three and four-loop self-energies could be derived from $\Phi_{4}$ and $\Phi_{5}$, respectively, but we choose not to give them here, since they are not needed for the set of four-loop vacuum diagrams that we will display explicitly in Sec. VII.

With Eqs. (60)-(62), the ring diagrams in Eq. (29) are readily written down.

\section{GENERIC MODEL ON THE LATTICE}

So far we have considered the generic model in Eq. (2). However, in a lattice regularization of gauge theories, higher vertices appear as well, without spoiling renormalizability. At the generic level, it is straightforward to add such couplings to the theory in Eq. (2). We can include, e.g., terms up to $\sim\left(1 / 8\right.$ !) $\gamma_{i j k l m n o p} \varphi_{i} \varphi_{j} \varphi_{k} \varphi_{l} \varphi_{m} \varphi_{n} \varphi_{o} \varphi_{p}$, as would arise in lattice perturbation theory for $\mathrm{SU}(N)$ gauge theories, if one keeps terms contributing to four-loop vacuum graphs. Such computations would be needed when one converts results of three-dimensional numerical Monte Carlo studies from lattice to continuum regularization [10].

In this case, everything goes as before, except for the appearance of extra vertices in the SD equations, as well as in Eq. (49). We shall here simply spell out the final results, without rewriting explicitly the modified SD equations. We obtain the following additional skeletons:

$$
\left.\Phi_{3}\right|_{\text {lat }}=\frac{1}{12}
$$


as well as the additional irreducible self-energy

$$
\left.\Pi_{2}^{\mathrm{irr}}\right|_{\text {lat }}=-\left(2-\left.\right|_{\text {lat }}=+\frac{1}{4} \cdot \bigcirc\right)+\frac{1}{4} \cdot Q+\frac{1}{6} \Omega+\frac{1}{8} \cdot \Omega \cdots
$$

where we again assumed $c_{i}=\frac{1}{2}$.

\section{APPLICATIONS: QCD, QED, SQED, ELECTROWEAK THEORY}

As an application of the generic formulas derived above, we consider in this section $\mathrm{SU}(N)$ gauge theory with fermions and a scalar field. This class includes QCD and QED (where graphs containing scalar propagators and, in the latter case, gauge field self-interactions are to be dropped out), as well as the electroweak theory and scalar electrodynamics (SQED). For brevity, we display here only the vertices appearing in the symmetric phases of the latter theories. We mostly use the language of QCD, referring to the gauge fields as gluons, etc.

The Lagrangian is specified by giving Feynman rules for the free propagators and free vertices,

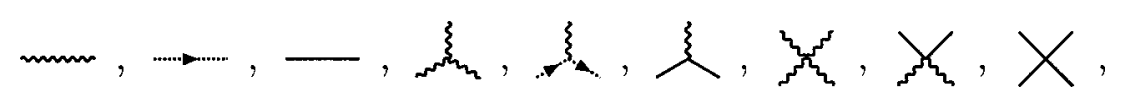

where gluons (scalars) are denoted by wavy (straight) lines. Both quarks and ghosts are denoted here by dotted lines; the Feynman rules for them are different, but the symmetry factors agree-the only exception being diagrams with more than one closed fermion loop, in which case both ghosts and quarks can appear in the same diagram simultaneously, reducing the symmetry by an obvious factor.

We do not here write down counterterms explicitly. Coupling constant counterterms can be viewed as a part of the cubic and quartic couplings, while wave function and mass counterterms can be treated as a part of the irreducible self-energies $\Pi_{n}^{\text {irr }}$, making their appearance only in ring diagrams according to Eq. (29).

Let us first note that once we write down the summation over the field content explicitly in Eq. (2), the "natural" symmetry factors in front of the vertices change. For instance, writing the four-point vertex in the case of two sets of fields, $\left\{\varphi_{i}\right\}$ $\rightarrow\left\{A_{i}\right\}+\left\{B_{\alpha}\right\}$, and using the symmetry of $\gamma_{i j k l}$, one gets

$$
\begin{aligned}
\frac{1}{4 !} \gamma_{i j k l} \varphi_{i} \varphi_{j} \varphi_{k} \varphi_{l}= & \frac{1}{4 !} \gamma_{i j k l} A_{i} A_{j} A_{k} A_{l}+\frac{1}{3 !} \gamma_{i j k \alpha} A_{i} A_{j} A_{k} B_{\alpha} \\
& +\frac{1}{(2 !)^{2}} \gamma_{i j \alpha \beta} A_{i} A_{j} B_{\alpha} B_{\beta}+\cdots
\end{aligned}
$$

Similarly, writing the three-point vertex for three different fields, $\left\{\varphi_{i}\right\} \rightarrow\left\{A_{i}\right\}+\left\{B_{\alpha}\right\}+\left\{C_{M}\right\}$, one finds

$$
\begin{aligned}
\frac{1}{3 !} \gamma_{i j k} \varphi_{i} \varphi_{j} \varphi_{k}= & \frac{1}{3 !} \gamma_{i j k} A_{i} A_{j} A_{k}+\frac{1}{2 !} \gamma_{i j \alpha} A_{i} A_{j} B_{\alpha} \\
& +\gamma_{i \alpha M} A_{i} B_{\alpha} C_{M}+\cdots
\end{aligned}
$$

With these conventions, each tree-level vertex in the graphical notation corresponds just to $\gamma_{i j k l}, \gamma_{i j k \alpha}$, etc., without any symmetry factors there: all of them are shown explicitly.
The only thing remaining is to write the summation over particle species explicitly also in the propagators of Eqs. (56)-(58),

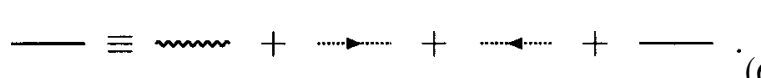

Only the vertices allowed by the Feynman rules are kept after this substitution. This generates all the graphs, with the correct symmetry factors.

\section{A. Vacuum skeletons up to the four-loop level}

The procedure outlined above can easily be carried out explicitly, and up to the four-loop level even by hand. The main complication is again the identification of various equivalent topologies, and for this a suitable algebraic notation may be more useful than a graphical one. As a result, for the field content in Eq. (66), we finally obtain 


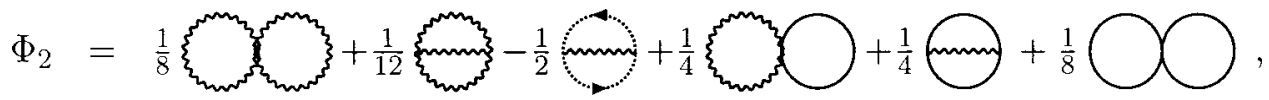

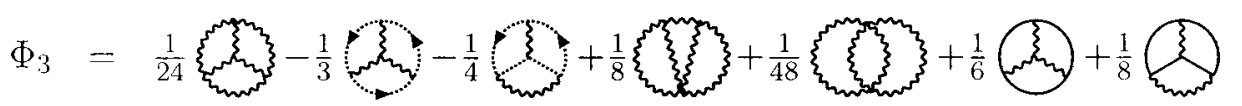

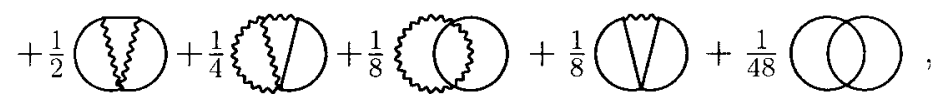

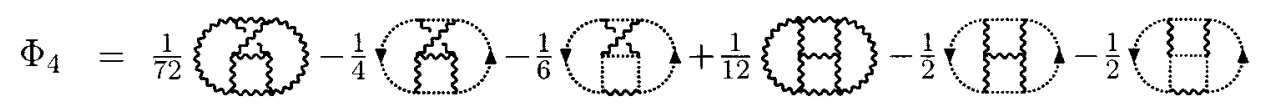

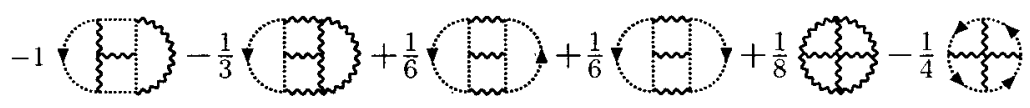

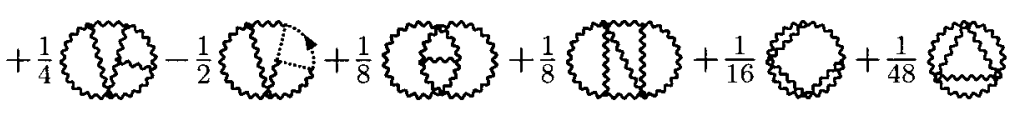

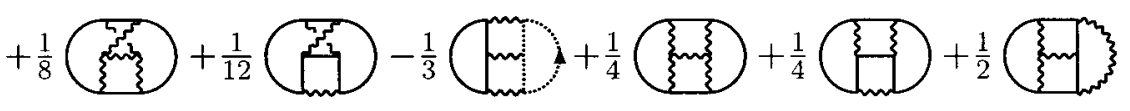

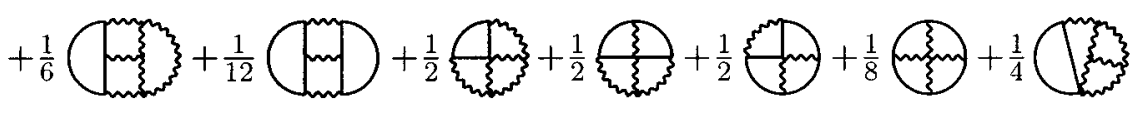

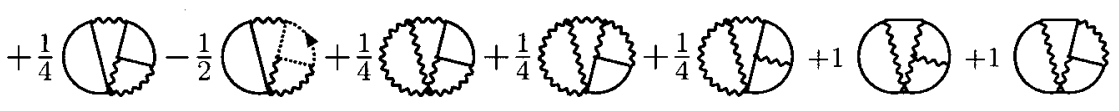

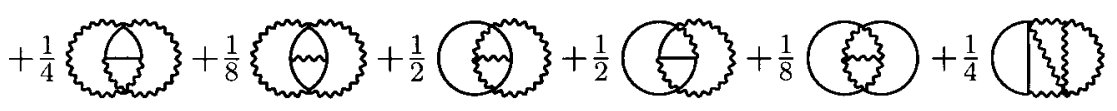

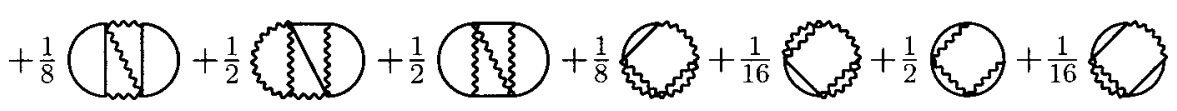

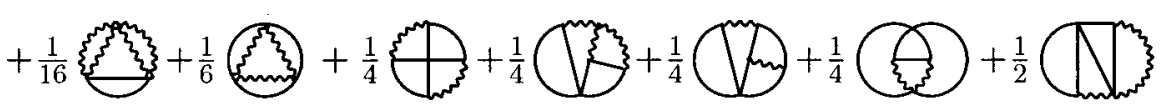

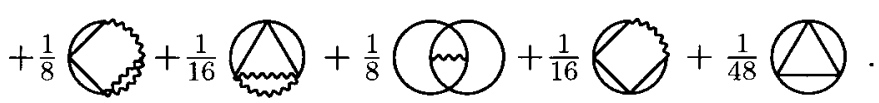

\section{B. Self-energies up to the two-loop level}

Using Eqs. (27), (20), the skeletons above immediately produce the self-energies of the model in Eq. (66). We obtain

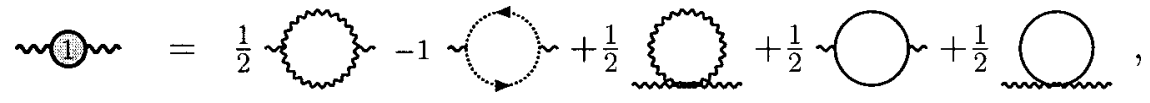

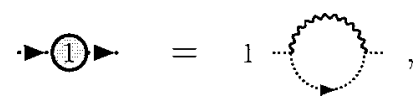

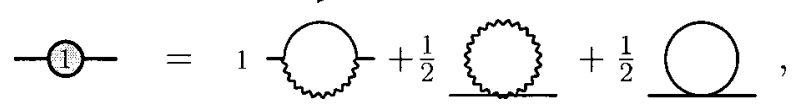

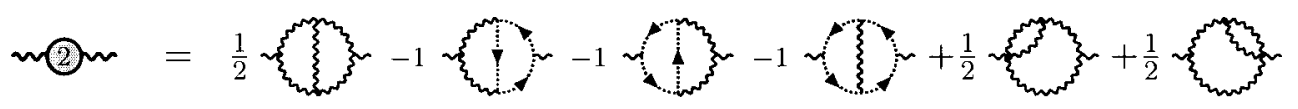

$$
\begin{aligned}
& +\frac{1}{4} \text { ข }
\end{aligned}
$$

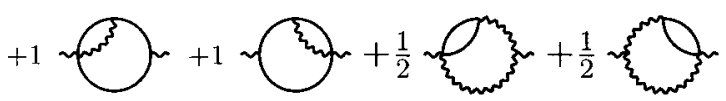

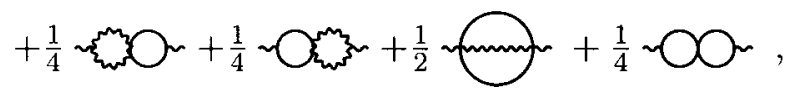




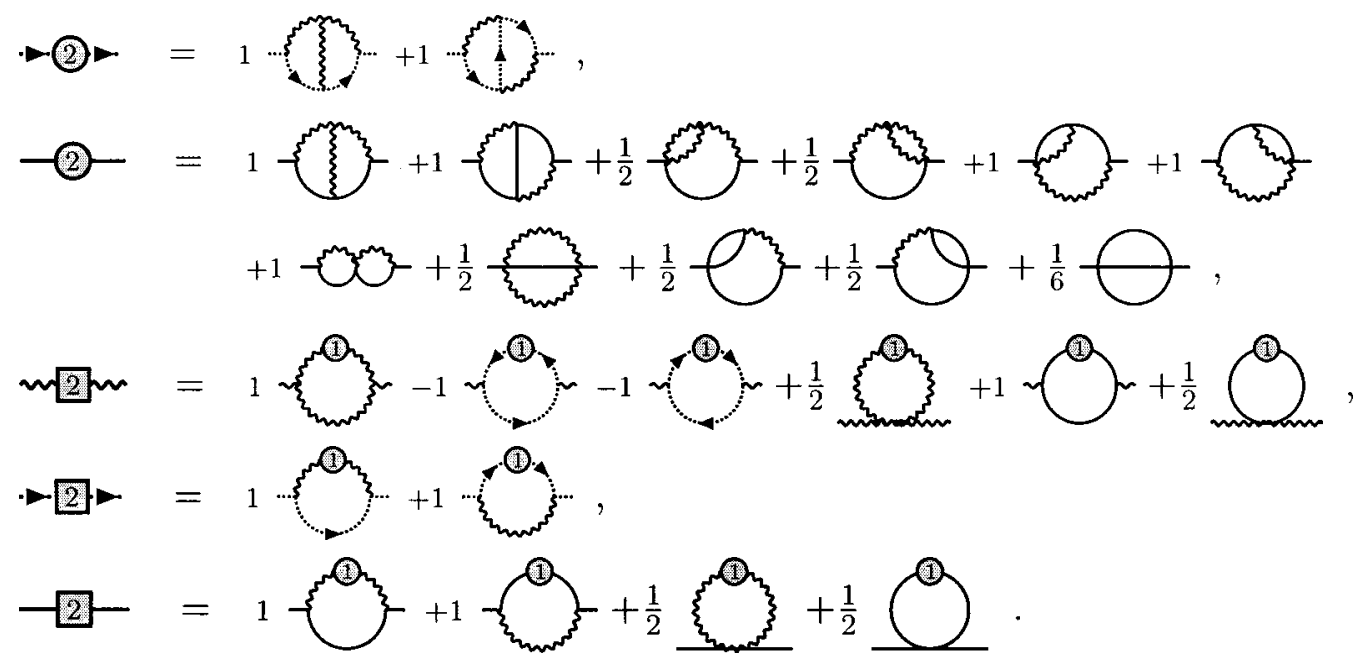

\section{Ring diagrams up to the four-loop level}

To be exhaustive up to the four-loop level, let us finally give the set of ring diagrams for the model of Eq. (66). While there are no ring diagrams up to the two-loop level, from Eq. (29) we get

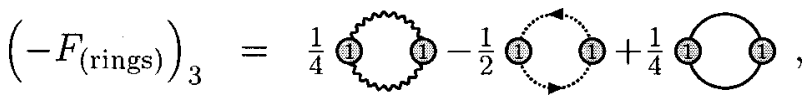

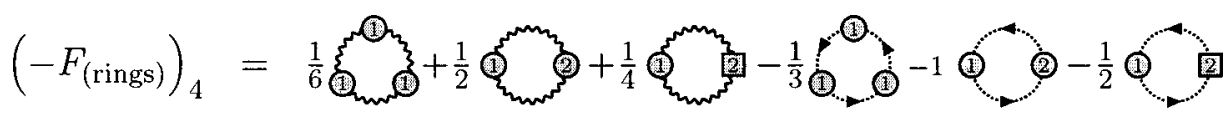

$$
\begin{aligned}
& +\frac{1}{6} \widehat{1}_{1}+\frac{1}{2} \text { (1) }+\frac{1}{4} \text { (1) }
\end{aligned}
$$

Note the extremely economic structure of the skeleton expansion of Eq. (29): the few ring diagrams above summarize 22 (276) three-loop (four-loop) diagrams.

\section{DISCUSSION}

In this paper we have described a simple practical procedure for systematically generating all vacuum diagrams of a given loop order in a generic field theory.

We have shown that the sum of vacuum diagrams can be written in the form of a modified skeleton expansion, Eq. (29). It contains two-particle-irreducible "skeletons" with free propagators, as well as various self-energy insertions inside "ring diagrams." The self-energies are, in turn, determined by the skeletons. Thus, all one really needs is the skeletons.

The two-particle-irreducible skeletons of a given order are, then, generated by Eq. (49). It contains a number of full three-point and four-point vertices, which can in turn be expanded using specific "irreducible" Schwinger-Dyson equations [Eqs. (40), (41), etc.], where full propagators have been replaced with free propagators. In this way, all vacuum graphs are generated simultaneously, with the correct symmetry factors. Finally, the precise particle content of the theory one is interested in can be specified as discussed in Sec. VII. Our method is also directly applicable to theories with spontaneous symmetry breaking, as only free propagators and vertices are modified; tadpole graphs are generated by Eq. (35).

This iterative procedure is very straightforward and can be automatized, but up to the four-loop level the computations are easily carried out even by hand, as we have demonstrated. Thus, we believe that our setup economizes the generation of the set of high-order vacuum diagrams, compared with techniques where all types of graphs have to be dealt with on the same footing, without a separation into skeletons with free propagators and ring diagrams.

Up to this point, we have not discussed at all the integrations remaining to be carried out after the diagrams have been generated. Let us end by pointing out that our setup is beneficial as far as their structure is considered, as well, in dimensions lower than 4 [17].

The point is that low-dimensional field theories of the type in Eq. (2) are superrenormalizable. In fact, for $d=2,3$, only the two-point function suffers from ultraviolet divergences, as can be seen by simple power counting. Therefore the skeleton graphs, which by definition do not have any genuine two-point functions inside them, do not contain any 
ultraviolet divergences in subdiagrams. The ring diagrams, on the other hand, do have ultraviolet divergences in subdiagrams. Note, in particular, that since $\Pi_{n}^{\mathrm{irr}}, \Pi_{n}^{\mathrm{red}(m)}$ come with different symmetry factors in Eq. (29), the counterterms in $\Pi_{n}^{\text {irr }}$, which make the whole $\Pi_{n}$ finite, do not in general immediately cancel all the ultraviolet subdivergences of the ring diagrams.

Consequently, various ring diagram classes can contribute to the overall divergences of the vacuum graphs with potentially infrared sensitive coefficients, coming from the other parts of the final integration, while skeleton diagrams cannot. Fortunately, the ring diagram integrations are simpler than those in the skeleton graphs, and this problem can thus be dealt with in a tractable setting [18].

\section{ACKNOWLEDGMENTS}

We thank M. Achhammer, U. Heinz, S. Leupold, and H. Schulz for useful discussions and correspondence. This work was partly supported by the TMR network Finite Temperature Phase Transitions in Particle Physics, EU Contract No. FMRX-CT97-0122, by the RTN network Supersymmetry and the Early Universe, EU Contract No. HPRN-CT-200000152, and by the Academy of Finland, Project No. 163065.
[1] V. W. Hughes and T. Kinoshita, Rev. Mod. Phys. 71, S133 (1999); A. Czarnecki and W. J. Marciano, Nucl. Phys. B (Proc. Suppl.) 76, 245 (1999).

[2] T. van Ritbergen, J. A. Vermaseren, and S. A. Larin, Phys. Lett. B 400, 379 (1997); K. G. Chetyrkin, ibid. 404, 161 (1997); J. A. Vermaseren, S. A. Larin, and T. van Ritbergen, ibid. 405, 327 (1997).

[3] K. G. Chetyrkin, A. L. Kataev, and F. V. Tkachov, Phys. Lett. 99B, 147 (1981); 101B, 457(E) (1981); K. G. Chetyrkin, S. G. Gorishnii, S. A. Larin, and F. V. Tkachov, ibid. 132B, 351 (1983); H. Kleinert, J. Neu, V. Schulte-Frohlinde, K. G. Chetyrkin, and S. A. Larin, Phys. Lett. B 272, 39 (1991); 319, 545(E) (1991); B. Kastening, Phys. Rev. D 57, 3567 (1998); S. A. Larin, M. Monnigmann, M. Strosser, and V. Dohm, Phys. Rev. B 58, 3394 (1998).

[4] E. Braaten and A. Nieto, Phys. Rev. Lett. 76, 1417 (1996).

[5] K. Kajantie, M. Laine, K. Rummukainen, and M. Shaposhnikov, Nucl. Phys. B503, 357 (1997).

[6] P. Arnold and C. Zhai, Phys. Rev. D 50, 7603 (1994); 51, 1906 (1995); C. Zhai and B. Kastening, ibid. 52, 7232 (1995); E. Braaten and A. Nieto, ibid. 53, 3421 (1996).

[7] A. D. Linde, Phys. Lett. 96B, 289 (1980).

[8] P. Ginsparg, Nucl. Phys. B170, 388 (1980); T. Appelquist and R. D. Pisarski, Phys. Rev. D 23, 2305 (1981).

[9] K. Kajantie, M. Laine, K. Rummukainen, and Y. Schröder, Phys. Rev. Lett. 86, 10 (2001).

[10] K. Farakos, K. Kajantie, K. Rummukainen, and M. Shaposhnikov, Nucl. Phys. B442, 317 (1995).

[11] M. Laine, Nucl. Phys. B451, 484 (1995); M. Laine and A. Rajantie, ibid. B513, 471 (1998).

[12] G. D. Moore, Nucl. Phys. B493, 439 (1997); B523, 569 (1998).
[13] R. Harlander and M. Steinhauser, Prog. Part. Nucl. Phys. 43, 167 (1999).

[14] J. Küblbeck, M. Böhm, and A. Denner, Comput. Phys. Commun. 60, 165 (1990); T. Hahn, ibid. 140, 418 (2001); http:// www.feynarts.de/

[15] P. Nogueira, J. Comput. Phys. 105, 279 (1993); ftp:// gtae2.ist.utl.pt/pub/qgraf/

[16] M. Bachmann, H. Kleinert, and A. Pelster, Phys. Rev. D 61, 085017 (2000); H. Kleinert, A. Pelster, B. Kastening, and M. Bachmann, Phys. Rev. E 62, 1537 (2000); B. Kastening, ibid. 61, 3501 (2000); H. Kleinert, A. Pelster, and B. Van den Bossche, hep-th/0107017; A. Pelster, H. Kleinert, and M. Bachmann, hep-th/0109014.

[17] M. Achhammer, Ph.D. thesis, University of Regensburg, 2000.

[18] K. Kajantie, M. Laine, K. Rummukainen, and Y. Schröder (in preparation).

[19] P. Cvitanović, Field Theory, Nordita Lecture Notes (Nordita, Copenhagen, 1983); http://www.nbi.dk/ predrag/field_the see also P. Cvitanović, B. Lautrup, and R. B. Pearson, Phys. Rev. D 18, 1939 (1978).

[20] J. M. Luttinger and J. C. Ward, Phys. Rev. 118, 1417 (1960); G. Baym, ibid. 127, 1391 (1962); C. De Dominicis and P. C. Martin, J. Math. Phys. 5, 31 (1964).

[21] J. M. Cornwall, R. Jackiw, and E. Tomboulis, Phys. Rev. D 10, 2428 (1974).

[22] J. Reinbach and H. Schulz, Phys. Lett. B 404, 291 (1997).

[23] R. Jackiw, Phys. Rev. D 9, 1686 (1974); R. Fukuda and E. Kyriakopoulos, Nucl. Phys. B85, 354 (1975).

[24] S. Coleman and E. Weinberg, Phys. Rev. D 7, 1888 (1973).

[25] J. A. M. Vermaseren, math-ph/0010025; http: //www.nikhef.nl/ form/ 